\title{
Existence of solutions for quasilinear elliptic equations with superlinear nonlinearities
}

\author{
Jia Gao*, Huang Lina and Zhang Xiaojuan
}

"Correspondence: gaojia79@yahoo.com.cn; jiagao2012@163.com College of Science, University of Shanghai for Science and Technology, Shanghai, 200093, China

\begin{abstract}
Working in a weighted Sobolev space, a new result involving superlinear nonlinearities for a quasilinear elliptic boundary value problem in a domain in $R^{N}$ is established. The proofs rely on the Galerkin method, Brouwer's theorem and a new weighted compact Sobolev-type embedding theorem due to V.L. Shapiro.
\end{abstract}

MSC: $35 J 25 ; 35 J 62 ; 65 L 60$

Keywords: weighted Sobolev space; superlinear; quasilinear elliptic equation

\section{Introduction}

Consider the following quasilinear elliptic problem:

$$
\left\{\begin{array}{l}
\mathcal{M} u=\left[\lambda_{1} u+f(x, u)\right] \rho-G, \quad x \in \Omega, \\
u \in H_{p, q, \rho}^{1}(\Omega, \Gamma),
\end{array}\right.
$$

where $\Omega$ is an open (possibly unbounded) set in $R^{N}(N \geq 1), \lambda_{1}$ is the first eigenvalue of $\mathcal{L}$ ((2.3) below), and $\mathcal{M}$ is a singular quasilinear elliptic operator defined by

$$
\mathcal{M} u=-\sum_{i, j=1}^{N} D_{i}\left[p_{i}^{\frac{1}{2}}(x) p_{j}^{\frac{1}{2}}(x) \sigma_{i}^{\frac{1}{2}}(u) \sigma_{j}^{\frac{1}{2}}(u) b_{i j}(x) D_{j} u\right]+b_{0} \sigma_{0} q u .
$$

The nonlinear part $f(x, u)$ in Eq. (1.1) satisfies certain superlinear conditions.

There have been many results for quasilinear elliptic equations under the conditions of which the nonlinearities satisfy sublinear or linear growth in a weighted Sobolev space. One can refer to [1-6].

However, there seem to be relatively few papers that consider the quasilinear elliptic equations with superlinearity, because the compactly embedding theorem cannot be obtained easily.

The aim of this paper is to obtain an existence result for problem (1.1). Our methods combine the Galerkin-type techniques, Brouwer's fixed-point theorem, and a new compactly embedding theorem established by V.L. Shapiro in [7].

This paper is organized as follows. In Section 2, we introduce some necessary assumptions and main results. In Section 3, four fundamental lemmas are established. In Section 4, the proofs of the main results are given.

\section{Springer}

(c) 2012 Gao et al.; licensee Springer. This is an Open Access article distributed under the terms of the Creative Commons Attribution License (http://creativecommons.org/licenses/by/2.0), which permits unrestricted use, distribution, and reproduction in any medium, provided the original work is properly cited. 


\section{Assumptions and main results}

In this section, we introduce some assumptions and give the main results in this paper.

Let $\Gamma \subset \partial \Omega$ be a fixed closed set (it may be the empty set) and $\rho(x), p_{i}(x), q(x) \in C^{0}(\Omega)$ be weight functions. $q(x)$ is nonnegative (maybe identically zero). Denote $p(x)$ as the vector function $\left(p_{1}(x), p_{2}(x), \ldots, p_{N}(x)\right)$.

Consider the following pre-Hilbert spaces

$$
C_{\rho}^{0}(\Omega)=\left\{\left.u \in C^{0}(\Omega)\left|\int_{\Omega}\right| u\right|^{2} \rho<\infty\right\}
$$

with inner product $\langle u, v\rangle_{\rho}=\int_{\Omega} u v \rho, \forall u, v \in C_{\rho}^{0}(\Omega)$, and

$$
\begin{aligned}
C_{p, q, \rho}^{1}(\Omega, \Gamma)= & \left\{u \in C^{0}(\bar{\Omega}) \cap C^{2}(\Omega) \mid u(x)=0, \forall x \in \Gamma ;\right. \\
& \left.\int_{\Omega}\left[\sum_{i=1}^{N}\left|D_{i} u\right|^{2} p_{i}+u^{2}(q+\rho)\right]<\infty\right\}
\end{aligned}
$$

with the inner product

$$
\langle u, v\rangle_{p, q, \rho}=\int_{\Omega}\left(\sum_{i=1}^{N} p_{i} D_{i} u D_{i} v+(q+\rho) u v\right)
$$

$\forall u, v \in C_{p, q, \rho}^{1}(\Omega)$ where $D_{i} u=\frac{\partial u}{\partial x_{i}}, i=1, \ldots, N$. Let $L_{\rho}^{2}(\Omega)$ be the Hilbert space obtained through the completion of $C_{\rho}^{0}(\Omega)$ by using the method of Cauchy sequences with respect to the norm $\|u\|_{\rho}=\langle u, u\rangle_{\rho}^{\frac{1}{2}}$, and $H_{p, q, \rho}^{1}(\Omega, \Gamma)$ be the completion of the space $C_{p, q, \rho}^{1}(\Omega, \Gamma)$ with the norm $\|u\|_{p, q, \rho}=\langle u, u\rangle_{p, q, \rho}^{\frac{1}{2}}$. Similarly, we may have $L_{p_{i}}^{2}(\Omega)(i=1, \ldots, N)$ and $L_{q}^{2}(\Omega)$. Consequently, (2.1) may lead to

$$
\langle u, v\rangle_{p, q, \rho}=\sum_{i=1}^{N}\left\langle D_{i} u, D_{i} v\right\rangle_{p_{i}}+\langle u, v\rangle_{\rho}+\langle u, v\rangle_{q}
$$

Definition 2.1 For the quasilinear differential operator $\mathcal{M}$, the two-form is

$$
\begin{aligned}
\mathcal{M}(u, v) & =\int_{\Omega} \sum_{i, j=1}^{N} p_{i}^{\frac{1}{2}} p_{j}^{\frac{1}{2}} \sigma_{i}^{\frac{1}{2}}(u) \sigma_{j}^{\frac{1}{2}}(u) b_{i j}(x) D_{j} u D_{i} v+\left\langle b_{0} \sigma_{0}(u) u, v\right\rangle_{q}, \\
\forall u, v & \in H_{p, q, \rho}^{1}(\Omega, \Gamma) .
\end{aligned}
$$

For the linear differential operator,

$$
\mathcal{L} u=-\sum_{i, j=1}^{N} D_{i}\left[p_{i}^{\frac{1}{2}} p_{j}^{\frac{1}{2}} a_{i j}(x) D_{j} u\right]+a_{0} q u
$$

the two-form is

$$
\mathcal{L}(u, v)=\int_{\Omega} \sum_{i, j=1}^{N} p_{i}^{\frac{1}{2}} p_{j}^{\frac{1}{2}} a_{i j}(x) D_{j} u D_{i} v+\left\langle a_{0} u, v\right\rangle_{q}, \quad \forall u, v \in H_{p, q, \rho}^{1}(\Omega, \Gamma) .
$$


Definition 2.2 $(\Omega, \Gamma)$ is a simple- $V_{L}$ region if the following conditions $\left(V_{L}-1\right)-\left(V_{L}-5\right)$ hold:

$\left(V_{L}-1\right)$ There exists a complete orthonormal system $\left\{\varphi_{n}\right\}_{n=1}^{\infty}$ in $L_{\rho}^{2}$. Also, $\varphi_{n} \in$ $H_{p, q, \rho}^{1}(\Omega, \Gamma) \cap C^{2}(\Omega), \forall n$;

$\left(V_{L}-2\right)$ There exists a sequence of eigenvalues $\left\{\lambda_{n}\right\}_{n=1}^{\infty}$, corresponding to the orthonormal sequence $\left\{\varphi_{n}\right\}_{n=1}^{\infty}$, and satisfying $0<\lambda_{1}<\lambda_{2} \leq \lambda_{3} \leq \cdots \leq \lambda_{n} \rightarrow \infty$ as $n \rightarrow \infty$, such that $\mathcal{L}\left(\varphi_{n}, v\right)=\lambda_{n}\left\langle\varphi_{n}, v\right\rangle_{\rho}, \forall v \in H_{p, q, \rho}^{1}(\Omega, \Gamma)$;

$\left(V_{L}-3\right) \Omega=\Omega_{1} \times \cdots \times \Omega_{N}$, where $\Omega_{i} \subset R$ is an open set for $i=1, \ldots, N$;

$\left(V_{L}-4\right)$ For each $p_{i}(x)$ and $\rho(x)$ in $\left(V_{L}-1\right)-\left(V_{L}-2\right)$, associated with each $\Omega_{i}$ there are positive functions $p_{i}^{*}(s), \rho_{i}^{*}(s) \in C^{0}\left(\Omega_{i}\right)$ satisfying $\int_{\Omega_{i}}\left[p_{i}^{*}(s)+\rho_{i}^{*}(s)\right] d s<\infty$, and $\rho(x)=$ $\rho_{1}^{*}\left(x_{1}\right) \cdots \rho_{N}^{*}\left(x_{N}\right), p_{i}(x)=\rho_{1}^{*}\left(x_{1}\right) \cdots \rho_{i-1}^{*}\left(x_{i-1}\right) p_{i}^{*}\left(x_{i}\right) \rho_{i+1}^{*}\left(x_{i+1}\right) \cdots \rho_{N}^{*}\left(x_{N}\right)$, for $i=1, \ldots, N$;

$\left(V_{L}-5\right)$ For each $\Omega_{i}, p_{i}^{*}, \rho_{i}^{*}(i=1, \ldots, N)$, there exists $h_{i} \in C^{0}\left(\Omega_{i}\right) \cap L_{\rho_{i}^{*}}^{\theta}\left(\Omega_{i}\right)$ for $2<\theta<\infty$ with the property

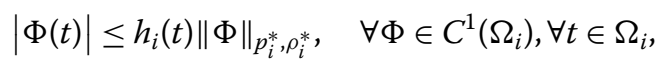

where $\|\Phi\|_{p_{i}^{*}, \rho_{i}^{*}}^{2}=\int_{\Omega_{i}}\left[p_{i}^{*}(t)\left|\frac{d \Phi(t)}{d t}\right|^{2}+\rho_{i}^{*}(t) \Phi^{2}(t)\right] d t$.

There are many examples to illustrate the Simple- $V_{L}$ region. One can refer to [7] and [8].

Remark 2.1 From $\left(V_{L}-3\right)$ and $\left(V_{L}-4\right)$, it is easy to see that $\rho(x), p_{i}(x)$ are positive and

$$
\int_{\Omega} \rho(x) d x<\infty, \quad \int_{\Omega} p_{i}(x) d x<\infty, \quad i=1, \ldots, N .
$$

Definition 2.3 $\mathcal{M}$ is near-related to $\mathcal{L}$ if the following condition holds:

$$
\lim _{\|u\|_{p, q, \rho} \rightarrow \infty} \frac{\mathcal{M}(u, v)-\mathcal{L}(u, v)}{\|u\|_{p, q, \rho}}=0, \quad \text { uniformly for }\|v\|_{p, q, \rho} \leq 1 .
$$

We make the following assumptions concerning the operators $\mathcal{M}$ and $\mathcal{L}: a_{i j}(x)(i, j=$ $1, \ldots, N)$ and $a_{0}(x)$ satisfy (so do $b_{i j}(x)$ and $b_{0}(x)$ ):

$$
\left\{\begin{array}{l}
\text { (1) } a_{0}(x), \quad a_{i j}(x) \in C^{0}(\Omega) \cap L^{\infty}(\Omega), i, j=1, \ldots, N \\
\text { (2) } a_{i j}(x)=a_{j i}(x), \quad \forall x \in \Omega, i, j=1, \ldots, N \\
\text { (3) } a_{0}(x) \geq \beta_{0}>0 \quad\left(b_{0}(x) \geq \beta_{1}>0\right), \quad \forall x \in \Omega ; \\
\text { (4) } \sum_{i, j=1}^{N} a_{i j}(x) \xi_{i} \xi_{j} \geq c_{0}|\xi|^{2} \quad\left(\sum_{i, j=1}^{N} b_{i j}(x) \xi_{i} \xi_{j} \geq c_{1}|\xi|^{2}\right), \\
\forall x \in \Omega, \xi \in R^{N} \text { where } c_{0}>0\left(c_{1}>0\right),|\xi|^{2}=\sum_{i=1}^{N} \xi_{i}^{2} .
\end{array}\right.
$$

It is assumed throughout the paper that $\sigma_{i}(u)(i=0,1, \ldots, N)$ meets:

$(\sigma-1) \sigma_{i}(u): H_{p, q, \rho}^{1}(\Omega, \Gamma) \rightarrow R$ is weakly sequentially continuous;

$(\sigma-2) \exists \eta_{0}, \eta_{1}>0$, s.t. $\eta_{0} \leq \sigma_{i}(u) \leq \eta_{1}, \forall u \in H_{p, q, \rho}^{1}(\Omega, \Gamma)$.

$f(x, s)$ meets the following conditions:

$(f-1) f(x, s)$ satisfies the Caratheodory conditions;

$(f-2)$ (superlinear growth condition) There exists $\theta$ with $2<\theta<\frac{2 N}{N-1}$, such that

$$
|f(x, s)| \leq h_{0}(x)+K|s|^{\theta-1}, \quad \forall s \in R \text {, a.e. } x \in \Omega,
$$

where $h_{0}(x) \in L_{\rho}^{\theta^{*}}(\Omega)$. $K$ is a nonnegative constant and $\theta^{*}=\frac{\theta}{\theta-1}$. 
$(f-3)$ There exists a nonnegative function $\widetilde{h}_{0}(x) \in L_{\rho}^{\theta^{*}}(\Omega)$ and a constant $\beta^{*}>0$, such that

$$
s f(x, s) \leq-\beta^{*}|s|^{2}+\widetilde{h}_{0}(x)|s|, \quad \forall s \in R \text {, a.e. } x \in \Omega .
$$

Remark 2.2 Observing that for $N=2, f(x, s)=-g(x) s|s|^{\frac{5}{3}}-\beta^{*}$, where $g(x) \in C^{0}(\Omega) \cap$ $L^{\infty}(\Omega)$ is a positive function, and meets both $(f-2)$ and $(f-3)$.

Now we state our main results in this paper.

Theorem 2.1 Assume that $(\Omega, \Gamma)$ is a simple- $V_{L}$ region, the operator $\mathcal{M}$ satisfies $(\sigma-1)$ $(\sigma-2)$, and is near-related to the operator $\mathcal{L},(2.5)$ holds for both $\mathcal{M}$ and $\mathcal{L}, f$ meets $(f-1)$ $(f-3)$, and $G \in\left[H_{p, q, \rho}^{1}(\Omega, \Gamma)\right]^{\prime}$ (the dual of $H_{p, q, \rho}^{1}(\Omega, \Gamma)$ ). Then the problem (1.1) has at least one nontrivial weak solution, that is, there exists $a u^{*} \in H_{p, q, \rho}^{1}(\Omega, \Gamma)$ such that

$$
\mathcal{M}\left(u^{*}, v\right)=\lambda_{1}\left\langle u^{*}, v\right\rangle_{\rho}+\int_{\Omega} f\left(x, u^{*}\right) v \rho-G(v) \quad \forall v \in H_{p, q, \rho}^{1}(\Omega, \Gamma) .
$$

To derive out Theorem 2.1, we first discuss the problem in $S_{n}$, which is the subspace of $H_{p, q, \rho}^{1}(\Omega, \Gamma)$ spanned by $\varphi_{1}, \ldots, \varphi_{n}$. Then by virtue of the Galerkin method, the results will be extended to $H_{p, q, \rho}^{1}(\Omega, \Gamma)$.

\section{Fundamental lemmas}

In this section, we introduce and establish four fundamental lemmas. Lemmas 3.1 and 3.2 give two useful embedding theorems. Lemma 3.3 constructs some approximation solutions in $S_{n}$. Lemma 3.4 studies the properties of the approximation solutions.

Lemma 3.1 ([7]) Assume that $\mathcal{L}$ is given by (2.3) and $(\Omega, \Gamma)$ is a simple- $V_{L}$ region. For $N \geq 2$, then $H_{p, q, \rho}^{1}(\Omega, \Gamma)$ is compactly imbedded in $L_{\rho}^{\theta}(\Omega)$ for $\forall \theta\left(2<\theta<\frac{2 N}{N-1}\right)$; for $N=1$, then $H_{p, q, \rho}^{1}(\Omega, \Gamma)$ is compactly imbedded in $L_{\rho}^{\theta}(\Omega)$ for $\forall \theta(2<\theta<\infty)$.

Lemma $3.2([7])$ Assume that $\mathcal{L}$ is given by $(2.3)$ and $(\Omega, \Gamma)$ is a simple- $V_{L}$ region. Then $H_{p, q, \rho}^{1}(\Omega, \Gamma)$ is compactly imbedded in $L_{\rho}^{2}(\Omega)$.

Lemma 3.3 Let all the assumptions in Theorem 2.1 hold. Then for $n \geq 2$, there exists a $u_{n} \in S_{n}$ such that

$$
\mathcal{M}\left(u_{n}, v\right)=\left(\lambda_{1}-\frac{1}{n}\right)\left\langle u_{n}, v\right\rangle_{\rho}+\int_{\Omega} f\left(x, u_{n}\right) v \rho-G(v), \quad \forall v \in S_{n} .
$$

Proof For fixed $n(n \geq 2)$ and $\forall \alpha=\left(\alpha_{1}, \ldots, \alpha_{n}\right) \in R^{n}$, set $u=\sum_{k=1}^{n} \alpha_{k} \varphi_{k}$. From simple- $V_{L}$ conditions, we obtain

$$
\begin{aligned}
& \mathcal{L}(u, u)=\sum_{k=1}^{n} \lambda_{k} \alpha_{k}^{2}, \quad\|u\|_{\rho}^{2}=\sum_{k=1}^{n} \alpha_{k}^{2}=|\alpha|^{2}, \\
& \mathcal{L}(u, u)+\|u\|_{\rho}^{2} \leq\left(\lambda_{n}+1\right)\|u\|_{\rho}^{2} .
\end{aligned}
$$


From (3) and (4) of (2.5), we have

$$
\mathcal{L}(u, u)+\|u\|_{\rho}^{2} \geq c_{0} \int_{\Omega} \sum_{i=1}^{N} p_{i}\left|D_{i} u\right|^{2}+\beta_{0}\|u\|_{q}^{2}+\|u\|_{\rho}^{2} \geq l\|u\|_{p, q, \rho}^{2},
$$

where $l=\min \left\{c_{0}, \beta_{0}, 1\right\}>0$. Combining (3.3) with (3.4), we get

$$
\|u\|_{\rho}^{2} \leq\|u\|_{p, q, \rho}^{2} \leq \frac{\lambda_{n}+1}{l}\|u\|_{\rho}^{2}
$$

For $m \geq 2$, a positive integer, we put

$$
f_{m}(x, s)= \begin{cases}f(x, m), & m \leq s \\ f(x, s), & -m \leq s \leq m ; \\ f(x,-m), & s \leq-m .\end{cases}
$$

Note from $(f-2)$ that $\left|f_{m}(x, s)\right| \leq h_{0}(x)+K|m|^{\theta-1}$ for $\forall s \in R$, a.e. $x \in \Omega$. Also, from $h_{0}(x) \in$ $L_{\rho}^{\theta^{*}}$, the Hölder inequality, Minkowski inequality, and (2.4), for $\forall v \in S_{n}$, we get

$$
\int_{\Omega}\left|f_{m}(x, u) v \rho\right| \leq\left\|f_{m}(x, u)\right\|_{L_{\rho}^{\theta^{*}}}\|v\|_{L_{\rho}^{\theta}} \leq T_{m}\|v\|_{L_{\rho}^{\theta}}
$$

where $T_{m}$ is a positive constant depending on $m$.

The remaining proof is separated into two parts. The first part is to prove the claim (3.8) for $f_{m}(x, s)$. The second part is to get the conclusion by leaving $m \rightarrow \infty$ based on (3.8).

Part 1. Fix $m(m \geq 2)$. To show there exists $u_{n, m}^{*}$ such that

$$
\mathcal{M}\left(u_{n, m}^{*}, v\right)=\left(\lambda_{1}-\frac{1}{n}\right)\left\langle u_{n, m}^{*}, v\right\rangle_{\rho}+\int_{\Omega} f_{m}\left(x, u_{n, m}^{*}\right) v \rho-G(v), \quad \forall v \in S_{n}
$$

we set

$$
F_{k}(\alpha)=\mathcal{M}\left(u, \varphi_{k}\right)-\left(\lambda_{1}-\frac{1}{n}\right)\left\langle u, \varphi_{k}\right\rangle_{\rho}-\int_{\Omega} f_{m}(x, u) \varphi_{k} \rho+G\left(\varphi_{k}\right), \quad k=1, \ldots, n .
$$

It is clear that $\sum_{k=1}^{n} F_{k}(\alpha) \alpha_{k}=I(\alpha)+I I(\alpha)$, where

$$
\begin{aligned}
& I(\alpha)=\mathcal{M}(u, u)-\mathcal{L}(u, u)-\int_{\Omega} f_{m}(x, u) u \rho+G(u), \\
& I I(\alpha)=\mathcal{L}(u, u)-\left(\lambda_{1}-\frac{1}{n}\right)\langle u, u\rangle_{\rho} .
\end{aligned}
$$

For (3.9), observing the fact that the operator $\mathcal{M}$ is near-related to $\mathcal{L}, G \in\left[H_{p, q, \rho}^{1}(\Omega, \Gamma)\right]^{\prime}$, (3.2), (3.5), (3.7), and Lemma 3.1, we conclude that

$$
\lim _{|\alpha| \rightarrow \infty} \frac{\mathcal{M}(u, u)-\mathcal{L}(u, u)}{|\alpha|^{2}}=0, \quad \lim _{|\alpha| \rightarrow \infty} \frac{\left|\int_{\Omega} f_{m}(x, u) u \rho\right|}{|\alpha|^{2}}=0, \quad \lim _{|\alpha| \rightarrow \infty} \frac{|G(u)|}{|\alpha|^{2}}=0,
$$

and

$$
\lim _{|\alpha| \rightarrow \infty} \frac{|I(\alpha)|}{|\alpha|^{2}}=0 .
$$


For (3.10), by (3.2), we obtain

$$
I I(\alpha)=\sum_{k=1}^{n}\left(\lambda_{k}-\lambda_{1}+\frac{1}{n}\right) \alpha_{k}^{2} \geq \frac{1}{n}|\alpha|^{2} .
$$

Consequently, $\sum_{k=1}^{n} F_{k}(\alpha) \alpha_{k} \geq \frac{|\alpha|^{2}}{2 n}$ where $|\alpha| \geq s_{0}$ (here $s_{0}$ is a large enough constant). By virtue of the generalized Brouwer's theorem [9], there exists $\gamma_{n, m}=\left(\gamma_{n, m}^{(1)}, \ldots, \gamma_{n, m}^{(n)}\right)$, such that $F_{k}\left(\gamma_{n, m}\right)=0, k=1, \ldots, n$. Taking $u_{n, m}^{*}=\sum_{k=1}^{n} \gamma_{n, m}^{(k)} \varphi_{k}$, then (3.8) holds.

Part 2. We claim that $\left\{\left\|u_{n, m}^{*}\right\|_{\rho}\right\}_{m=2}^{\infty}$ (n fixed) is uniformly bounded according to $m$.

Arguing by contradiction, without loss of generality, suppose that

$$
\lim _{m \rightarrow \infty}\left\|u_{n, m}^{*}\right\|_{\rho}=\infty
$$

Taking $v=u_{n, m}^{*}$ in (3.8),

$$
\begin{aligned}
& \mathcal{L}\left(u_{n, m}^{*}, u_{n, m}^{*}\right)-\lambda_{1}\left\langle u_{n, m}^{*}, u_{n, m}^{*}\right\rangle_{\rho}+\frac{1}{n}\left\langle u_{n, m}^{*}, u_{n, m}^{*}\right\rangle_{\rho} \\
& \quad=\int_{\Omega} f_{m}\left(x, u_{n, m}^{*}\right) u_{n, m}^{*} \rho-G\left(u_{n, m}^{*}\right)+\mathcal{L}\left(u_{n, m}^{*}, u_{n, m}^{*}\right)-\mathcal{M}\left(u_{n, m}^{*}, u_{n, m}^{*}\right)
\end{aligned}
$$

holds, that is,

$$
\begin{aligned}
& \sum_{k=1}^{n}\left(\lambda_{k}-\lambda_{1}\right)\left|\widehat{u}_{n, m}^{*}(k)\right|^{2}+\frac{1}{n}\left\|u_{n, m}^{*}\right\|_{\rho}^{2} \\
& \quad=\int_{\Omega} f_{m}\left(x, u_{n, m}^{*}\right) u_{n, m}^{*} \rho-G\left(u_{n, m}^{*}\right)+\mathcal{L}\left(u_{n, m}^{*}, u_{n, m}^{*}\right)-\mathcal{M}\left(u_{n, m}^{*}, u_{n, m}^{*}\right),
\end{aligned}
$$

where $\widehat{u}_{n, m}^{*}(k)=\left\langle\varphi_{k}, u_{n, m}^{*}\right\rangle_{\rho}$.

On the other hand, using $(f-3)$, for $s \geq m$, we have

$$
s f_{m}(x, s)=\frac{s}{m} \cdot m f(x, m) \leq \widetilde{h}_{0}(x)|s|, \quad \text { a.e. } x \in \Omega
$$

Similarly, we can also obtain the same conclusion where $-m \leq s \leq m$ or $s \leq-m$. As a result,

$$
s f_{m}(x, s) \leq \widetilde{h}_{0}(x)|s|, \quad \forall s \in R \text {, a.e. } x \in \Omega
$$

(3.14) and (3.16) imply that

$$
\frac{1}{n}\left\|u_{n, m}^{*}\right\|_{\rho}^{2} \leq\left\|\widetilde{h}_{0}\right\|_{L_{\rho}^{\theta *}}\left\|u_{n, m}^{*}\right\|_{L_{\rho}^{\theta}}+\left|G\left(u_{n, m}^{*}\right)\right|+\mathcal{L}\left(u_{n, m}^{*}, u_{n, m}^{*}\right)-\mathcal{M}\left(u_{n, m}^{*}, u_{n, m}^{*}\right) .
$$

Dividing both sides of (3.17) by $\left\|u_{n, m}^{*}\right\|_{\rho}^{2}$ and leaving $m \rightarrow \infty$, we obtain from the fact that $\mathcal{M}$ is near-related to $\mathcal{L}, G \in\left[H_{p, q, \rho}^{1}(\Omega, \Gamma)\right]^{\prime}, \widetilde{h}_{0}(x) \in L_{\rho}^{\theta^{*}}(\Omega)$, and (3.5) together with Lemma 3.1 that $\frac{1}{n} \leq 0$. However, $n$ is a positive integer. So, we have arrived at a contradiction. (3.12) does not hold. Then

$$
\exists K_{1}>0, \quad\left\|u_{n, m}^{*}\right\|_{\rho} \leq K_{1}, \quad \forall m \geq 2 .
$$


(3.5) and (3.18) imply that there is a subsequence of $\left\{u_{n, m}^{*}\right\}_{m=2}^{\infty}$ (for ease of notation take the full sequence) and a $u_{n} \in S_{n}[10]$ such that

$$
\left\{\begin{array}{l}
\lim _{m \rightarrow \infty}\left\|u_{n, m}^{*}-u_{n}\right\|_{p, q, \rho}=0 ; \\
\lim _{m \rightarrow \infty} u_{n, m}^{*}(x)=u_{n}(x), \quad \text { a.e. } x \in \Omega ; \\
\lim _{m \rightarrow \infty} D_{i} u_{n, m}^{*}(x)=D_{i} u_{n}(x), \quad \text { a.e. } x \in \Omega, i=1, \ldots, N
\end{array}\right.
$$

Therefore, from (3.19), we obtain

$$
\lim _{m \rightarrow \infty} \mathcal{M}\left(u_{n, m}^{*}, v\right)=\mathcal{M}\left(u_{n}, v\right), \quad \forall v \in S_{n}
$$

And recall Lemma 3.1 that

$$
\lim _{m \rightarrow \infty} \int_{\Omega}\left|u_{n, m}^{*}-u_{n}\right|^{\theta} \rho=0
$$

Moreover, there exists a $W(x) \in L_{\rho}^{\theta}$ and a subsequence $\left\{u_{n, m_{j}}^{*}\right\}_{j=1}^{\infty} \subset\left\{u_{n, m}^{*}\right\}_{m=2}^{\infty}$ such that $\left|u_{n, m_{j}}^{*}(x)\right| \leq W(x)$, a.e. $x \in \Omega$ for $\forall j$.

Since by virtue of the Hölder inequality, $(f-1),(f-2)$ and the Lebesgue dominated convergence theorem, we get

$$
\lim _{j \rightarrow \infty} \int_{\Omega} f_{m_{j}}\left(x, u_{n, m_{j}}^{*}\right) v \rho=\int_{\Omega} f\left(x, u_{n}\right) v \rho, \quad \forall v \in S_{n} .
$$

Now replacing $m$ by $m_{j}$ in (3.8) and taking the limit as $j \rightarrow \infty$ on both sides of the equation, we consequently obtain that (3.1) holds and Lemma 3.3 is completed.

Lemma 3.4 Let all the assumptions in Theorem 2.1 hold. Then the sequence $\left\{u_{n}\right\}$ obtained in Lemma 3.3 is uniformly bounded in $H_{p, q, \rho}^{1}(\Omega, \Gamma)$.

Proof For $\left\{u_{n}\right\}_{n=2}^{\infty} \subset S_{n}$ in Lemma 3.3, set $u_{n}=\sum_{k=1}^{n} \widehat{u}_{n}(k) \varphi_{k}$ where $\widehat{u}_{n}(k)=\left\langle\varphi_{k}, u_{n}\right\rangle_{\rho}$.

We suppose that Lemma 3.4 is false. Without loss of generality, suppose that

$$
\lim _{n \rightarrow \infty}\left\|u_{n}\right\|_{p, q, \rho}=\infty
$$

To lead to a contradiction, taking $v=u_{n}$ in (3.1), then

$$
\mathcal{M}\left(u_{n}, u_{n}\right)=\left(\lambda_{1}-\frac{1}{n}\right)\left\langle u_{n}, u_{n}\right\rangle_{\rho}+\int_{\Omega} f\left(x, u_{n}\right) u_{n} \rho-G\left(u_{n}\right)
$$

And we can get

$$
\frac{1}{n}\left\|u_{n}\right\|_{\rho}^{2} \leq \int_{\Omega} f\left(x, u_{n}\right) u_{n} \rho+\left|G\left(u_{n}\right)\right|+\mathcal{L}\left(u_{n}, u_{n}\right)-\mathcal{M}\left(u_{n}, u_{n}\right)
$$

In view of $G \in\left[H_{p, q, \rho}^{1}(\Omega, \Gamma)\right]^{\prime},(f-3)$, and (3.24),

$$
\left(\frac{1}{n}+\beta^{*}\right)\left\|u_{n}\right\|_{\rho}^{2} \leq\left\|\tilde{h}_{0}\right\|_{L_{\rho}^{\theta^{*}}}\left\|u_{n}\right\|_{L_{\rho}^{\theta}}+\left|G\left(u_{n}\right)\right|+\mathcal{L}\left(u_{n}, u_{n}\right)-\mathcal{M}\left(u_{n}, u_{n}\right) .
$$


Dividing both sides of (3.25) by $\|u\|_{p, q, \rho}^{2}$ and leaving $n \rightarrow \infty$, from the fact that $\mathcal{M}$ is nearrelated to $\mathcal{L}, \widetilde{h}_{0}(x) \in L_{\rho}^{\theta^{*}}$ and Lemma 3.1, we get

$$
\lim _{n \rightarrow \infty} \frac{\left\|u_{n}\right\|_{\rho}}{\left\|u_{n}\right\|_{p, q, \rho}}=0
$$

Apply (3) and (4) of (2.5) in conjunction with $(\sigma-2)$ to show

$$
\mathcal{M}\left(u_{n}, u_{n}\right) \geq c_{1} \eta_{0} \int_{\Omega} \sum_{i=1}^{N} p_{i}\left|D_{i} u_{n}\right|^{2}+\beta_{1} \eta_{0}\left\|u_{n}\right\|_{q}^{2}
$$

also, (1) and (2) of (2.5) to show

$$
\mathcal{L}\left(u_{n}, u_{n}\right) \leq K_{2} \int_{\Omega} \sum_{i=1}^{N} p_{i}\left|D_{i} u_{n}\right|^{2}+K_{3}\left\|u_{n}\right\|_{q}^{2}
$$

where $K_{2}$ and $K_{3}$ are positive constants. Setting $c_{2}=\min \left\{c_{1} \eta_{0}, \beta_{1} \eta_{0}\right\}$ and $c_{3}=\max \left\{K_{2}, K_{3}\right\}$, it is obvious $c_{2}, c_{3}>0$. By (3.27) and (3.28), we conclude that

$$
\frac{c_{2}}{c_{3}} \mathcal{L}\left(u_{n}, u_{n}\right) \leq \mathcal{M}\left(u_{n}, u_{n}\right)
$$

Using (3.29) and $(f-3)$, from (3.25), we obtain

$$
\frac{c_{2}}{c_{3}} \mathcal{L}\left(u_{n}, u_{n}\right)+\beta^{*}\left\|u_{n}\right\|_{\rho}^{2} \leq \lambda_{1}\left\|u_{n}\right\|_{\rho}^{2}+\left\|\widetilde{h}_{0}\right\|_{L_{\rho}^{\theta *}}\left\|u_{n}\right\|_{L_{\rho}^{\theta}}+\left|G\left(u_{n}\right)\right| .
$$

From (3.4), we get $\mathcal{L}\left(u_{n}, u_{n}\right)+\left\|u_{n}\right\|_{\rho}^{2} \geq l\left\|u_{n}\right\|_{p, q, \rho}^{2}$. Set $c_{4}=\min \left\{\frac{c_{2}}{c_{3}}, \beta^{*}\right\}$. It is easy to obtain

$$
c_{4} l\left\|u_{n}\right\|_{p, q, \rho}^{2} \leq \lambda_{1}\left\|u_{n}\right\|_{\rho}^{2}+\left\|\tilde{h}_{0}\right\|_{L_{\rho}^{\theta^{*}}}\left\|u_{n}\right\|_{L_{\rho}^{\theta}}+\left|G\left(u_{n}\right)\right| .
$$

Dividing both sides of (3.31) by $\|u\|_{p, q, \rho}^{2}$ and leaving $n \rightarrow \infty$, by (3.26), we get

$$
c_{4} l \leq 0 .
$$

So, we have arrived at a contradiction. Thus, there holds

$$
\left\|u_{n}\right\|_{p, q, \rho} \leq K_{4}, \quad \forall n \geq 2 \text { and some } K_{4}>0 .
$$

Lemma 3.4 is completed.

\section{Proof of Theorem 2.1}

Proof Since $H_{p, q, \rho}^{1}(\Omega, \Gamma)$ is a separable Hilbert space, from Lemma 3.1 and Lemma 3.2, we conclude that there exist a subsequence of $\left\{u_{n}\right\}_{n=2}^{\infty}$ (which for ease of notation we take the full sequence) and a function $u^{*} \in H_{p, q, \rho}^{1}(\Omega, \Gamma)$ with the following properties [10]:

$$
\lim _{n \rightarrow \infty}\left[\left\|u_{n}-u^{*}\right\|_{\rho}+\int_{\Omega}\left|u_{n}-u^{*}\right|^{\theta} \rho\right]=0
$$




$$
\begin{aligned}
& \exists W^{\prime}(x) \in L_{\rho}^{2}(\Omega) \cap L_{\rho}^{\theta}(\Omega), \quad \text { s.t. }\left|u_{n}(x)\right| \leq W^{\prime}(x), \text { a.e. } x \in \Omega, n \geq 2 ; \\
& \lim _{n \rightarrow \infty} u_{n}(x)=u^{*}(x), \quad \text { a.e. } x \in \Omega ; \\
& \lim _{n \rightarrow \infty}\left\langle D_{i} u_{n}, v\right\rangle_{p_{i}}=\left\langle D_{i} u^{*}, v\right\rangle_{p_{i}}, \quad \forall v \in L_{p_{i}}^{2}, i=1, \ldots, N ; \\
& \lim _{n \rightarrow \infty}\left\langle u_{n}, v\right\rangle_{q}=\left\langle u^{*}, v\right\rangle_{q}, \quad \forall v \in L_{q}^{2} ; \\
& \lim _{n \rightarrow \infty} G\left(u_{n}\right)=G\left(u^{*}\right) .
\end{aligned}
$$

Let $v_{J} \in S_{J}$ where $J \geq 2$ is a fixed but arbitrary positive integer. In fact, for $n \geq J$, we have

$$
\begin{aligned}
\mathcal{M}\left(u_{n}, v_{J}\right)-\mathcal{M}\left(u^{*}, v_{J}\right)= & \int_{\Omega} \sum_{i, j=1}^{N}\left\{p_{i}^{\frac{1}{2}} p_{j}^{\frac{1}{2}} \sigma_{i}^{\frac{1}{2}}\left(u_{n}\right) \sigma_{j}^{\frac{1}{2}}\left(u_{n}\right) b_{i j}(x) D_{j}\left(u_{n}-u^{*}\right) D_{i} v_{J}\right. \\
& \left.+p_{i}^{\frac{1}{2}} p_{j}^{\frac{1}{2}}\left[\sigma_{i}^{\frac{1}{2}}\left(u_{n}\right) \sigma_{j}^{\frac{1}{2}}\left(u_{n}\right)-\sigma_{i}^{\frac{1}{2}}\left(u^{*}\right) \sigma_{j}^{\frac{1}{2}}\left(u^{*}\right)\right] b_{i j}(x) D_{j} u^{*} D_{i} v_{J}\right\} \\
& +\left\langle b_{0}\left[\sigma_{0}\left(u_{n}\right) u_{n}-\sigma_{0}\left(u^{*}\right) u^{*}\right], v_{J}\right\rangle_{q}
\end{aligned}
$$

Observing (1) of (2.5), $(\sigma-1)-(\sigma-2),(4.3)-(4.5)$ and $D_{i} v_{J} \in L_{p_{i}}^{2}$, we have

$$
\lim _{n \rightarrow \infty} \mathcal{M}\left(u_{n}, v_{J}\right)=\mathcal{M}\left(u^{*}, v_{J}\right)
$$

On the other hand, applying $(f-1),(f-2),(4.2),(4.3)$, the Hölder inequality, and the Lebesgue dominated convergence theorem, we obtain that

$$
\lim _{n \rightarrow \infty} \int_{\Omega} f\left(x, u_{n}\right) v_{J} \rho=\int_{\Omega} f\left(x, u^{*}\right) v_{J} \rho, \quad \text { a.e. } x \in \Omega \text {. }
$$

Also, by (3.1), we have

$$
\mathcal{M}\left(u_{n}, v_{J}\right)=\left(\lambda_{1}-\frac{1}{n}\right)\left\langle u_{n}, v_{J}\right\rangle_{\rho}+\int_{\Omega} f\left(x, u_{n}\right) v_{J} \rho-G\left(v_{J}\right) .
$$

For (4.10), leaving $n \rightarrow \infty$, from (4.1), (4.8), and (4.9), we have

$$
\mathcal{M}\left(u^{*}, v_{J}\right)=\lambda_{1}\left\langle u^{*}, v_{J}\right\rangle_{\rho}+\int_{\Omega} f\left(x, u^{*}\right) v_{J} \rho-G\left(v_{J}\right)
$$

Next, given $v \in H_{p, q, \rho}^{1}(\Omega, \Gamma)$, we define a projection $P_{J}: H_{p, q, \rho}^{1}(\Omega, \Gamma) \rightarrow S_{J}$, that is,

$$
P_{J} v=\sum_{k=1}^{J} \widehat{v}(k) \varphi_{k} \in S_{J}
$$

where $\widehat{v}(k)=\left\langle\varphi_{k}, v\right\rangle_{\rho}$. It is easy to get $\lim _{j \rightarrow \infty}\left\|P_{J} v-v\right\|_{p, q, \rho}=0$. As a result, there hold

$$
\left\{\begin{array}{l}
\lim _{J \rightarrow \infty} \mathcal{M}\left(u^{*}, P_{J} v\right)=\mathcal{M}\left(u^{*}, v\right), \\
\lim _{J \rightarrow \infty}\left\langle u^{*}, P_{J} v\right\rangle_{\rho}=\left\langle u^{*}, v\right\rangle_{\rho}, \\
\lim _{J \rightarrow \infty} \int_{\Omega} f\left(x, u^{*}\right) P_{J} v \rho=\int_{\Omega} f\left(x, u^{*}\right) v \rho, \\
\lim _{J \rightarrow \infty} G\left(P_{J} v\right)=G(v) .
\end{array}\right.
$$


Replacing $v_{J}$ by $P_{J} v$ in (4.11), passing to the limit as $J \rightarrow \infty$ on both sides, and using (4.13), we can obtain

$$
\mathcal{M}\left(u^{*}, v\right)=\lambda_{1}\left\langle u^{*}, v\right\rangle_{\rho}+\int_{\Omega} f\left(x, u^{*}\right) v \rho-G(v), \quad \forall v \in H_{p, q, \rho}^{1}(\Omega, \Gamma) .
$$

Hence, the proof of Theorem 2.1 is complete.

\section{Competing interests}

The authors declare that they have no competing interests.

\section{Authors' contributions}

The paper is the result of joint work of all authors who contributed equally to the final version of the paper. All authors read and approved the final manuscript.

\section{Acknowledgements}

The authors express their sincere thanks to the referees for their valuable suggestions. This work was supported financially by the National Natural Science Foundation of China (11171220).

Received: 23 March 2012 Accepted: 6 August 2012 Published: 10 August 2012

\section{References}

1. Berestycki, H, Figueredo, DG: Double resonance in semilinear elliptic problems. Commun. Partial Differ. Equ. 6(1), 91-120 (1981)

2. Rumbos, A, Shapiro, VL: Jumping nonlinearities and weighted Sobolev spaces. J. Differ. Equ. 214, 326-357 (2005)

3. Rumbos, A: A semilinear elliptic boundary value problem at resonance where the nonlinearity may grow linearly. Nonlinear Anal. TMA 16, 1159-1168 (1991)

4. Lefton, L, Shapiro, VL: Resonance and quasilinear parabolic differential equations. J. Differ. Equ. 101, 148-177 (1993)

5. Shapiro, VL: Resonance, distributions and semilinear elliptic partial differential equations. Nonlinear Anal. TMA 8, 857-871 (1984)

6. Jia, G, Zhao, Q: Existence results in weighted Sobolev spaces for some singular quasilinear elliptic equations. Acta Appl. Math. 109, 599-607 (2010)

7. Shapiro, VL: Singular Quasilinearity and Higher Eigenvalues. Memoirs of the American Mathematical Society, vol. 726. Am. Math. Soc., Providence (2001)

8. Shapiro, VL: Special functions and singular quasilinear partial differential equations. SIAM J. Math. Anal. 22, 1411-1429 (1991)

9. Kesavan, S: Topics in Functional Analysis and Applications. Wiley, New York (1989)

10. Xuan, BJ: Variational Methods. University of Science and Technology of China Press, Hefei (2006)

doi:10.1186/1687-2770-2012-90

Cite this article as: Gao et al.: Existence of solutions for quasilinear elliptic equations with superlinear nonlinearities. Boundary Value Problems 2012 2012:90.

\section{Submit your manuscript to a SpringerOpen ${ }^{\circ}$ journal and benefit from:}

- Convenient online submission

- Rigorous peer review

- Immediate publication on acceptance

- Open access: articles freely available online

- High visibility within the field

- Retaining the copyright to your article 